\title{
Cross-reactivity between six Enterobacteriaceae complete lipopolysaccharide core chemotypes
}

\author{
NDUBISI A. NNALUE, G. NASSER KHAN and NADA MUSTAFA \\ Department of Medical Microbiology, Faculty of Medicine and Health Sciences, United Arab Emirates \\ University, PO Box 17666, Al Ain, United Arab Emirates
}

\begin{abstract}
To gain insight into the value of lipopolysaccharide (LPS) core determinants for crossprotective immunisation the serological relationships between six complete (LPS) core types from Enterobacteriaceae were investigated. Hyperimmune sera were raised in mice by repeated immunisation with heat-killed strains of Salmonella choleraesuis (Ra core type) or Escherichia coli (core types R1, R2, R3, R4 and K12) and characterised for reactivity with complete and incomplete core chemotypes by ELISA and immunoblotting. Three sera (anti-Ra, anti-R2 and anti-R3) reacted strongly with 3-5 different complete core types whereas the other three (anti-R1, anti-R4 and anti-K12) reacted strongly only with their homologous core types in these assays. Two approaches were used to examine further the structural bases for cross-reactivity between these cores. By the first approach the anti-complete-core sera were tested for cross-reactivity with truncated forms of the Salmonella species core (incomplete cores) derived from coredefective mutants. By the second approach, antisera raised against some core-defective mutants were tested for cross-reactivity with complete cores. The results of these investigations revealed that several pair-wise combinations of core types can be used as immunogens to elicit immune responses that recognise all six core types and that the major determinants which mediate cross-reactivity between complete cores are localised in the outer core region.
\end{abstract}

\section{Introduction}

Enteric organisms cause various gut, urinary tract and systemic infections in man and animals including gastro-enteritis, septicaemia and typhoid fever. These infections and their complications such as septic shock are increasing in incidence and importance as a result of the increasing use of immunosuppressive therapies and the increasing numbers of elderly and debilitated individuals. The lipopolysaccharide (LPS), an amphipathic molecule anchored in the outer membrane of gram-negative bacteria, plays a pivotal role in many aspects of host-parasite interactions of enteric bacteria. As a virulence factor it protects the bacteria from destruction by complement and macrophages and as an endotoxin it acts as an agonist of host defences and

Received 9 June 1998; revised version accepted 18 Aug. 1998.

Corresponding author: Dr N. A. Nnalue. Present address: Tonna Bioservices and Consulting, 8813 Allman Rd, Lenexa, KS 66219, USA. triggers the release of inflammatory mediators by monocytes [1]. Being a strong immunogen, it also makes a highly accessible target for protective immune responses. In enteric organisms, the LPS comprises three distinct domains: the $\mathrm{O}$ specific polysaccharide chain ( $\mathrm{O}$ antigen), the core oligosaccharide (the core) and lipid $\mathrm{A}$ (Fig. 1). The $\mathrm{O}$ antigen, the outer and readily accessible domain of LPS, displays a high degree of structural heterogeneity while the core and lipid A domains are highly conserved.

While the need for cross-protective vaccines against enteric bacteria has long been recognised [2], the vaccines that are currently available elicit mainly typespecific protective responses directed against the $O$ antigen [3]. The large body of work on crossprotection by core determinants remains controversial in that some studies found that immunisation with rough strains cross-protected [4-7] while others did not [8-11]. However, recent clinical studies support the concept that anti-core immune responses can protect in natural settings by showing that natural levels of anti-endotoxin core antibodies (EndoCAb) correlate with better outcomes from infections and 

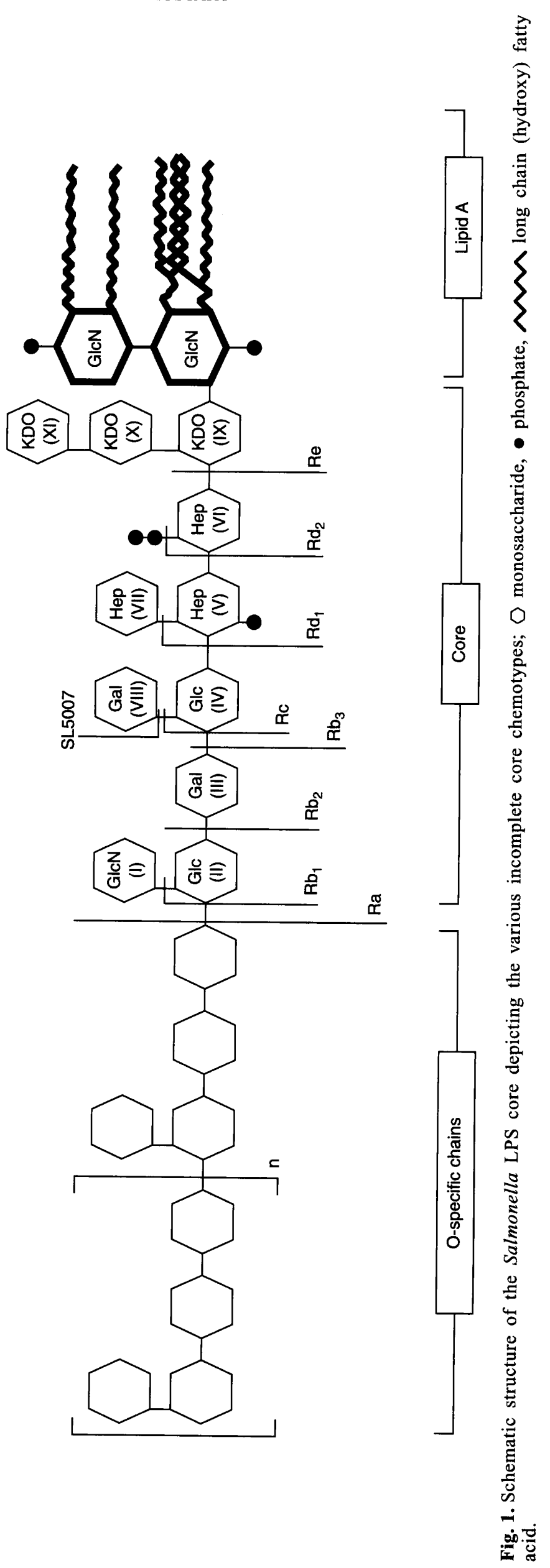
reduced incidence of complications after surgery [1216]. Furthermore, the accessibility of some core epitopes to recognition by antibodies has been demonstrated by immunochemical studies $[17,18]$.

The structures of the different core types (Fig. 2) found among enteric organisms have been well characterised [19-21], but little is known about serological relationships between them. A knowledge of such relationships is necessary for identification or construction of appropriate strains for use as crossprotective vaccines. The purpose of this study was to characterise cross-reactivity between the six complete core types found among Salmonella species and Escherichia coli to gain insight on the identity of cross-reactive core epitopes and to develop a rationale for cross-protective immunisation.

\section{Materials and methods}

\section{Bacterial strains}

The complete-core strains used included Salmonella choleraesuis strain SN57 (chemotype Ra), S. typhimurium strain TV119 (Ra) and E. coli strains HF4704 (R1), EH100 (R2), F653 (R3), F2513 (R4) and W3110 (K12). Strains with truncated cores include $S$. typhimurium strains SL733 $\left(\mathrm{Rb}_{1}\right)$, TV161 $\left(\mathrm{Rb}_{2}\right)$, TV148 $\left(\mathrm{Rb}_{3}\right)$, SL805 (Rc), SL1032 $\left(\mathrm{Rd}_{1}\right)$, SL1181 $\left(\mathrm{Rd}_{2}\right)$, SL1102 (Re) and SL5007. The LPS from strain SL5007 (hereby designated $\mathrm{Rb}_{4}$ ) has not been previously assigned a chemotype designation and differs in structure from the Ra core only because it lacks the Gal $1 \rightarrow 6$ branch residue (VII in Fig. 2). These strains

Ra

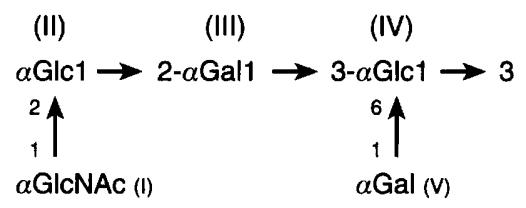

R1

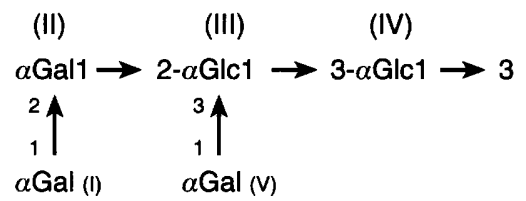

$\mathrm{R} 2$

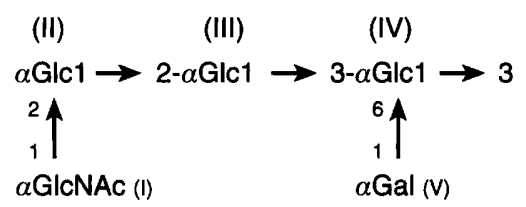

have all been described previously [20-23]. L-broth (per L: tryptone $10 \mathrm{~g}$, yeast extract $5 \mathrm{~g}, \mathrm{NaCl} 10 \mathrm{~g}$ ), Oxoid blood agar base (code CM55) or MacConkey agar (Difco) were used as appropriate for batch cultivation of these organisms. Tryptone-yeast extract broth (per L: tryptone $10 \mathrm{~g}$, yeast extract $5 \mathrm{~g}, \mathrm{NH}_{4} \mathrm{Cl}$ $2.5 \mathrm{~g}, \mathrm{Na}_{2} \mathrm{HPO}_{4}, 15 \mathrm{~g}, \mathrm{KH}_{2} \mathrm{PO}_{4} 6 \mathrm{~g}, \mathrm{Na}_{2} \mathrm{SO}_{4} .10 \mathrm{H}_{2} \mathrm{O}$ $0.5 \mathrm{~g}$ ) supplemented with glucose $10 \mathrm{~g} / \mathrm{L}$ was used for cultivation by fermenter.

\section{LPS extraction and purification}

Bacteria were grown to late logarithmic phase with constant aeration in a 12-L fermenter (Pharmacia-LKB, Uppsala, Sweden). LPS was extracted from smooth strains by the phenol-water procedure [24] and from rough strains by the phenol-chloroform-petroleum ether method [25]. The resulting crude preparations were extensively treated with DNAase, RNAase and proteinase $\mathrm{K}$ to eliminate proteins and to reduce the level of nucleic acid contamination to $\leqslant 5 \%$. Purified LPS preparations were lyophilised and where necessary subjected to electrodialysis and conversion to the triethylamine salt forms to increase solubility in water [26].

\section{Preparation of heat-killed bacterial antigens}

Heat-killed suspensions were prepared from bacteria with special precautions to ensure purity and homogeneity. Strains were kept at $-80^{\circ} \mathrm{C}$ in a broth:glycerol 2:1 mixture and streaked out directly on agar (Oxoid blood agar base; code CM55) when needed for antigen preparation. Several colonies were picked from each

R3

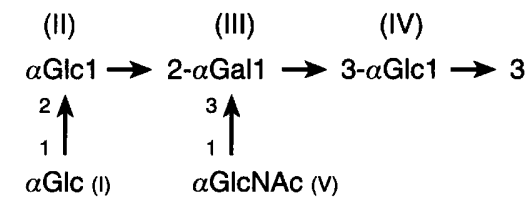

R4

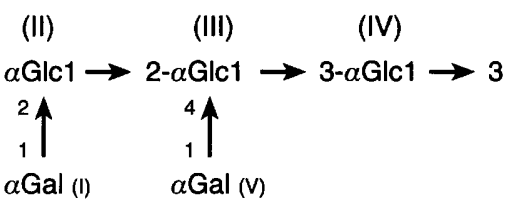

$\mathrm{K} 12$

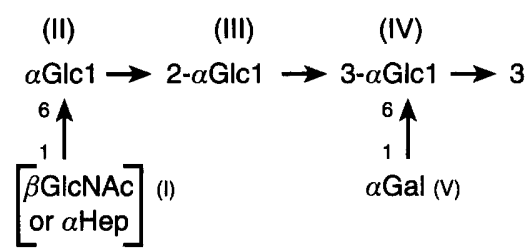

Fig. 2. Structure of the hexose regions of six complete core types found among Salmonella and E. coli strains. Roman numerals designate corresponding sugar residues. 
plate, resuspended in tryptic soy broth (TSB) and then spread on several plates of CM55. The plates were incubated overnight at $37^{\circ} \mathrm{C}$ and the resulting bacterial growth was scraped off and resuspended in $10 \mathrm{ml}$ of saline. Suspensions were washed twice by centrifugation and finally resuspended in $3 \mathrm{ml}$ of saline. They were kept overnight at $4^{\circ} \mathrm{C}$ while counts were determined by dilution plating; then they were adjusted to contain c. $2 \times 10^{10} \mathrm{cfu} / \mathrm{ml}$ and heated $\left(80^{\circ} \mathrm{C}, 3 \mathrm{~h}\right)$ to kill the bacteria. Heat-treated suspensions were tested for sterility by transfer of $50-\mu 1$ samples into $5 \mathrm{ml}$ of fresh TSB followed by overnight incubation at $37^{\circ} \mathrm{C}$.

\section{Preparation of antisera}

TO mice, a reasonably vigorous outbred stock, were originally purchased from Harlan Olac, London, and subsequently bred in the facilities at the Desert and Marine Environment Research Center, United Arab Emirates University. This stock was originally developed by Dr M. Theiler at the State Serum Institute, Denmark. Mice were divided into groups of 10 and bled for pre-immune serum 1 week before the start of immunisation. Antisera designated anti-Ra, anti-R1,

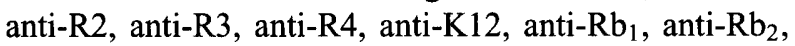
anti- $\mathrm{Rb}_{4}$, anti- $\mathrm{Rd}_{1}$, anti- $R \mathrm{~d}_{2}$, anti-Re were prepared by intraperitoneal (i.p.) inoculation of these mice with appropriate heat-killed strains. The immunisation schedule comprised six injections administered once per week. Immunisation was started with an initial dose of $10^{8}$ bacterial cells and doubled at each subsequent inoculation so that the last dose contained c. $3 \times 10^{9}$ heat-killed bacterial cells. Mice were bled after the fourth, fifth and sixth injections and sera from each group were pooled.

\section{Enzyme immunoassay ELISA}

Flat-bottomed EIA plates (Maxisorp, Nunc, Roskilde, Denmark), were coated with glycoconjugates in $0.05 \mathrm{M}$ carbonate buffer ( $\mathrm{pH}$. 9.6) or with LPS by chloroformethanol evaporation [27]. The plates were blocked by treatment for $1 \mathrm{~h}$ at $37^{\circ} \mathrm{C}$ with bovine serum albumin (BSA) $0.5 \%$ and gelatin $0.025 \%$ in $0.05 \mathrm{M}$ carbonate buffer, $\mathrm{pH}$. 9.6, and washed three times in washing buffer $(0.15 \mathrm{M} \mathrm{NaCl}$, Tween $20 \quad 0.05 \%)$. Aliquots $(100 \mu \mathrm{l})$ of the antisera were diluted in dilution buffer (Tween $200.05 \%$, BSA $0.5 \%$ and gelatin $0.025 \%$ in PBS) and $100 \mu \mathrm{l}$ volumes were added to the wells in duplicate followed by incubation for $90 \mathrm{~min}$ each at $37^{\circ} \mathrm{C}$ and at room temperature. Plates were washed as before and peroxidase-labelled rabbit anti-mouse polyvalent immunoglobulins (Dakopattis, Glostrup, Denmark) or goat anti-rabbit IgG (Sigma) as appropriate was diluted as recommended by the manufacturer and added to each well. The plates were incubated and washed again; then substrate solution (sodium phosphate buffer containing o-phenylenediamine $\mathrm{HCl}$ $1 \mathrm{mg} / \mathrm{ml}$ and $30 \% \mathrm{H}_{2} \mathrm{O}_{2} 1 \mu \mathrm{l} / \mathrm{ml}$ ) was added followed by incubation for $60 \mathrm{~min}$ at $37^{\circ} \mathrm{C}$. The reaction was stopped by the addition of $75 \mu \mathrm{l}$ of $2 \mathrm{M} \mathrm{H}_{2} \mathrm{SO}_{4}$ to each well and absorbance was read at $490 \mathrm{~nm}$ in a BioRad model 3550 EIA reader. The end-point titre (EPT) was the dilution of serum that gave an $\mathrm{A}_{490}$ value of 0.2 . The relative reactivity of each serum with each antigen was calculated as follows. EIA data from each titration assay were plotted on double logarithmic axes; then a region encompassing three successive dilutions at which all titration curves were linear or approximately so was delineated by visual inspection. The geometric mean absorbance $\left(\mathrm{Gm}_{\mathrm{ab}}\right)$ for the three dilutions in the region was determined for each antigen. The $\mathrm{Gm}_{\mathrm{ab}}$ for the homologous antigen (LPS from immunising strain) was assigned a relative reactivity value of $100 \%$. Values for other antigens were calculated by dividing their $\mathrm{Gm}_{\mathrm{ab}}$ by that of the homologous antigen and multiplying by 100 .

\section{SDS-PAGE and immunoblotting}

LPS samples $(2.5 \mu \mathrm{g})$ were electrophoresed in polyacrylamide $15 \%$ gels incorporating SDS and urea as described previously [28]. They were transferred electrophoretically $(120 \mathrm{~mA}, 12 \mathrm{~h})$ to nitrocellulose membranes and tested for reactivity with sera as described previously [17] with the same conjugate as in EIA but diaminobenzidine $/ \mathrm{H}_{2} \mathrm{O}_{2}$ (Sigma) as substrate system. For immunoblotting, antisera were added to membranes at concentrations 50-fold higher than the ELISA EPT with their respective homologous LPS. For dot immunoblotting LPS samples $(10 \mu \mathrm{g} / \mathrm{dot})$ were deposited on nitrocellulose membrane with a dot filtration manifold (Gibco/BRL). The membrane was then cut into strips as appropriate and tested for reactivity with sera at 10 -fold their EPT.

\section{Results}

Reactivity of anti-core sera with complete cores in ELISA

All six anti-core sera reacted strongly with their respective homologous LPS in ELISA, demonstrating the following EPTs: anti-Ra, 72900; anti-R1, 24300; anti-R2, 24 300; anti-R3, 72 900; anti-R4, 24300; and anti-K12, 24300 . They also reacted to different extents with the various heterologous LPS (data not shown) while none of the pre-immune sera did (EPT <300). The data from titration of two sera, anti-Ra and antiR3, are presented in Fig. 3 and are representative of results obtained with all six sera. The semi-logarithmic plots on the right clearly demonstrate approximately linear relationships between serum dilution and reactivity with LPS. This suggests that non-specific interactions contributed no more than minimally to reactivity despite the complex nature of reactants, LPS and unabsorbed antisera. The relative reactivities of all six anti-core sera with complete cores are shown in Fig. 4. Anti-Ra and anti-R2 manifested the broadest cross-reactivity, demonstrating strong $(\geqslant 50 \%)$ or mod- 


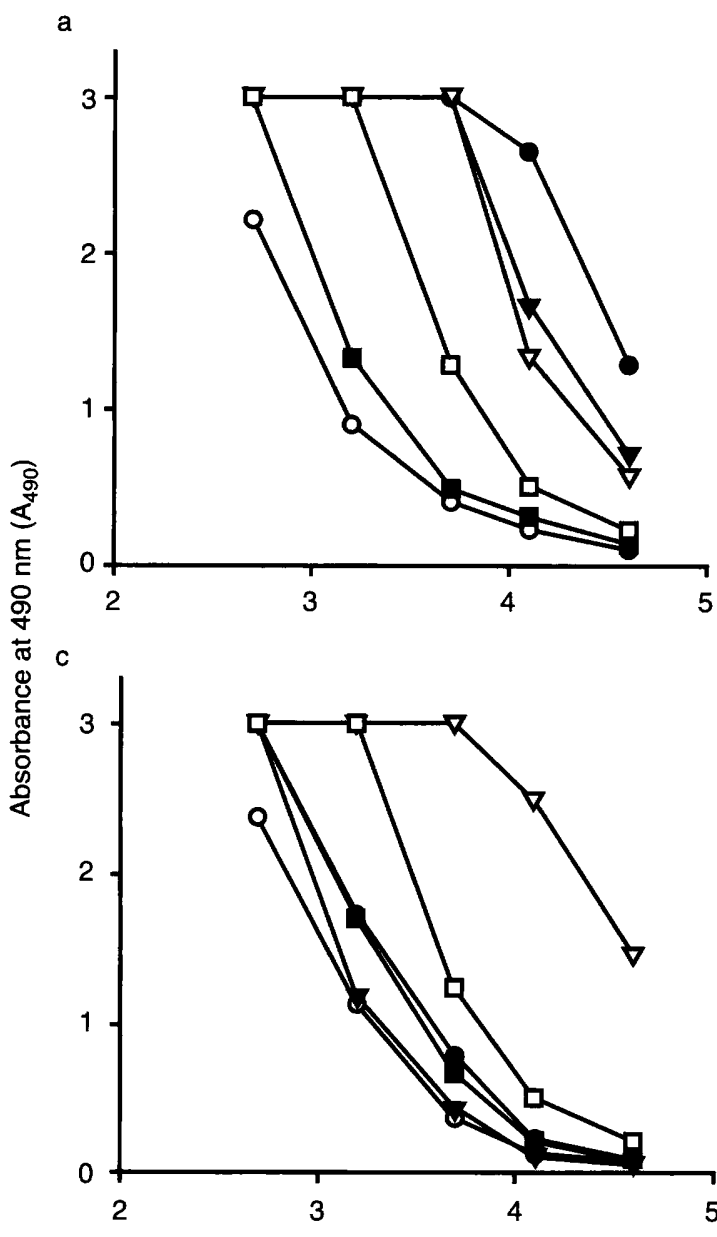

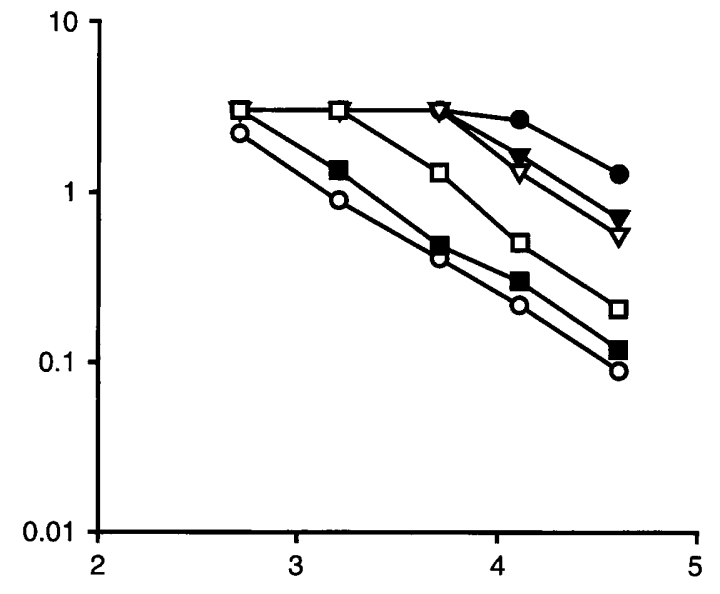

d

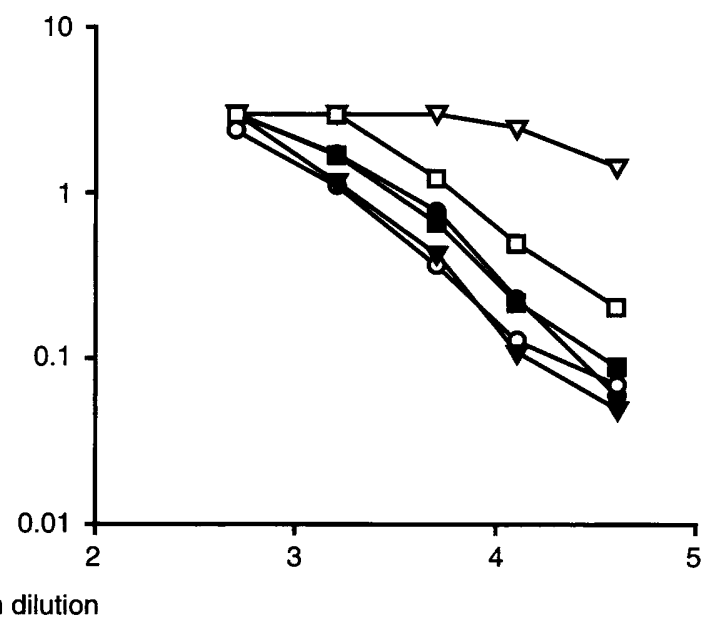

Fig. 3. Titration of two anti-core sera (anti-Ra, a and $\mathbf{b}$; anti-R3, $\mathbf{c}$ and d) against five complete core chemotypes. Symbols represent LPS of chemotypes $\mathrm{Ra}(\bullet), \mathrm{R} 1(0), \mathrm{R} 2(\boldsymbol{\nabla}), \mathrm{R} 3(\nabla), \mathrm{R} 4(\boldsymbol{\bullet}), \mathrm{K} 12(\mathrm{\square})$.

erate $(25-49 \%)$ reactivity with four complete cores. Anti-K12 reacted strongly with $\mathrm{K} 12$ and moderately with R2 LPS whereas the remaining three sera (anti$\mathrm{R} 1$, anti-R3 and anti-R4) reacted weakly $(\leqslant 25 \%)$ with all but their homologous LPS.

\section{Reactivity of anti-core sera with complete cores in dot immunoblots}

When tested by dot-blotting, most anti-core sera (anti$\mathrm{R} 1$, anti-R2, anti-R3 and anti-R4) reacted strongly with their homologous LPS only and cross-reacted weakly with no more than one other core type (Fig. 5). The exceptions were anti-Ra and anti-K12, both of which cross-reacted strongly with R2 LPS and showed a weak recognition for at least two other complete core types. It is of note that anti-R2 did not react with K12 LPS despite the strong recognition of R2 LPS by anti-K12.

\section{Reactivity of anti-core sera with electrophoretically resolved complete cores}

To confirm that the observed cross-reactivity of antisera was due to recognition of moieties corresponding to complete cores, the LPS samples were first separated by SDS-PAGE and transferred to nitrocellulose membranes before being tested for reactivity with sera. The results (Fig. 6) showed that the sera recognised only single bands of similar size among the different complete cores. However, the reactivity patterns differed somewhat from those obtained by dot-blotting. Some antisera (anti-Ra, anti-R2 and anti-R3) crossreacted more strongly or recognised more complete cores types than they did in dot-blots, while others (anti-R4 and anti-K12) recognised fewer core types. Notable among the former was anti-R3, which reacted with five complete cores, contrary to its narrow specificity in dot-blots. To investigate whether the use of different serum concentrations for transblots $(50 \times \mathrm{EPT})$ and dot-blots $(10 \times \mathrm{EPT})$ was responsible for the differential reactivity of anti-R3, this serum was tested in the former system at $10 \times \mathrm{EPT}$. At this higher serum dilution, anti R3 (anti-R3* in Fig. 6) recognised only R3 LPS, just as it did in dot-blots.

\section{Reactivity of anti-core sera with incomplete cores}

Four antisera (anti-Ra, anti-R2, anti-R3 and anti-K12) were tested for reactivity with various truncated derivatives of the $\mathrm{Ra}$ core to delineate the determinants 

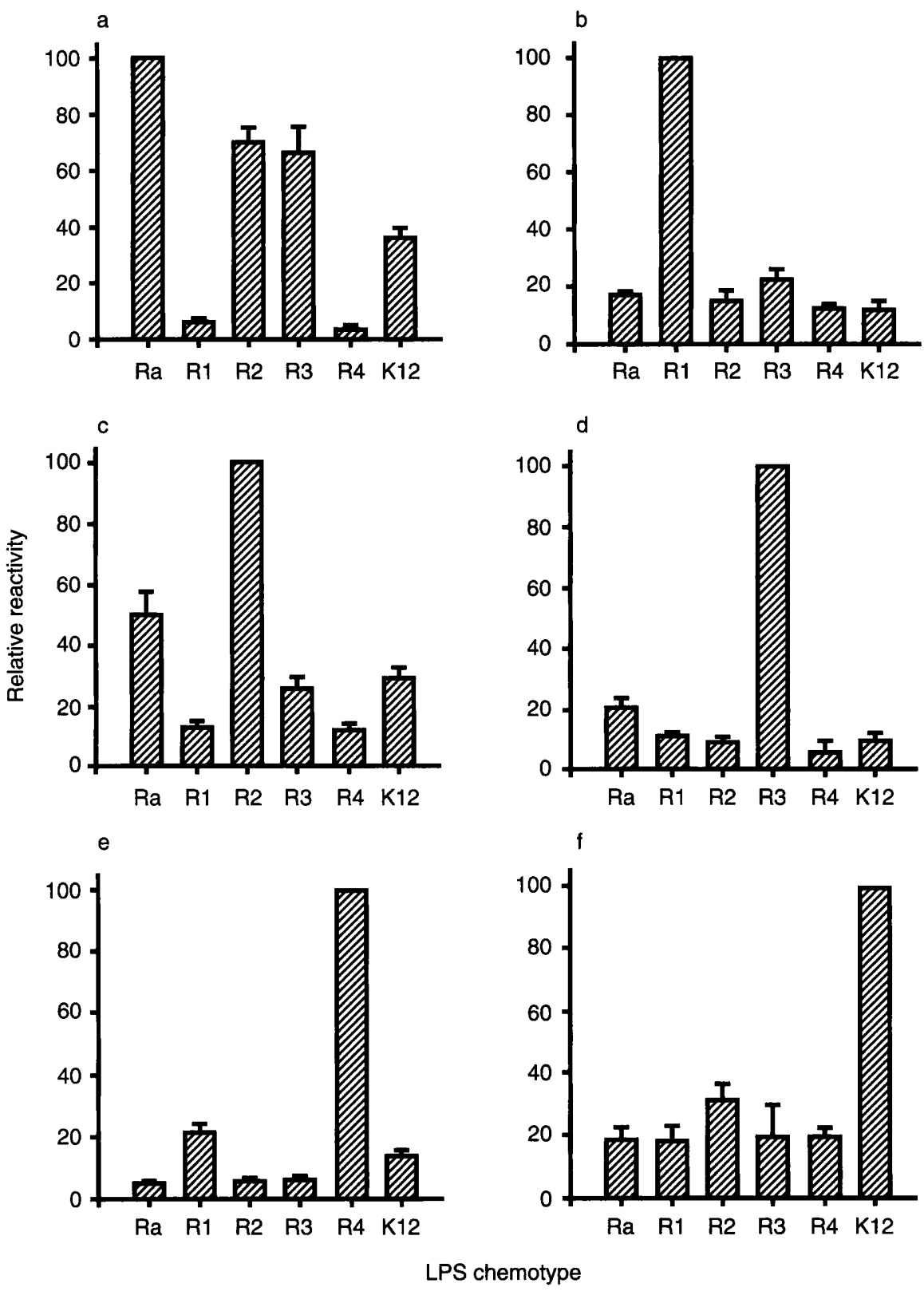

Fig. 4. Relative reactivity of anti-core sera with various complete core chemotypes determined by ELISA. Data represent mean and SEM of three determinations: a, anti-Ra; b, anti-R1; c, anti-R2; d, anti-R3; e, anti-R4; f, anti-K12.

involved in the cross-reactivity between their homologous LPS. All four sera reacted well with $R b_{1}$ and $R b_{2}$ LPS and also selectively recognised $R_{3}$ or $R c$ LPS, or both (Fig. 7). However, none reacted with $\mathrm{Rd}_{1}$, $\mathrm{Rd}_{2}$ and $\mathrm{Re}$ LPS. This showed that anti-core sera generated by immunisation with complete core strains lacked antibodies to inner core epitopes and crossreacted with complete cores based on antibodies directed at outer core epitopes.

\section{Reactivity of antisera generated against core-} defective mutants with complete cores

To directly compare the roles of inner and outer core epitopes in cross-reactivity between complete cores, antisera raised against selected incomplete core chemotypes were tested for reactivity with these cores. Anti-
$\mathrm{Rb}_{1}$ and anti- $\mathrm{Rb}_{2}$ (Fig. 8), raised against outer core chemotypes, cross-reacted with several complete cores whereas anti- $\mathrm{Rd}_{2}$ and anti-Re, raised against deep core chemotypes, failed to recognise even the $\mathrm{Ra}$ core. However, anti- $\mathrm{Rd}_{1}$, also raised against an inner core chemotype, recognised five complete cores, showing that some inner core epitopes are accessible and can mediate cross-reactivity between complete cores. Anti$\mathrm{Rb}_{4}$ recognised all six complete cores, including types $\mathrm{R} 1$ and R4, which were not recognised by anti-Ra.

\section{Discussion}

The need to develop vaccines that would cross-protect against the large number of enterobacterial serotypes has long been recognised and has been approached 


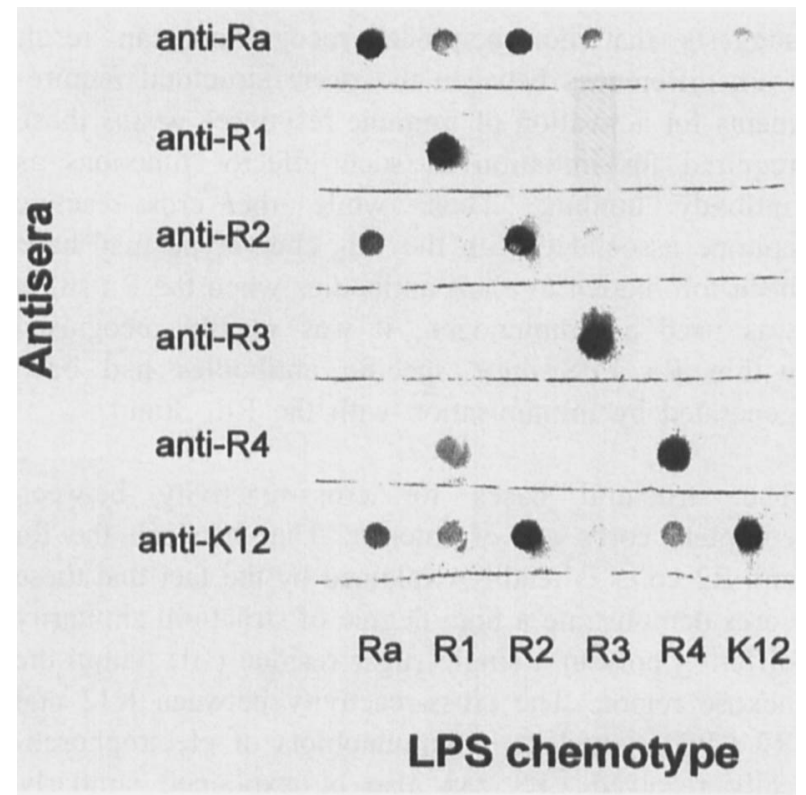

Fig. 5. Dot-immunoblotting of anti-core sera against complete core LPS.

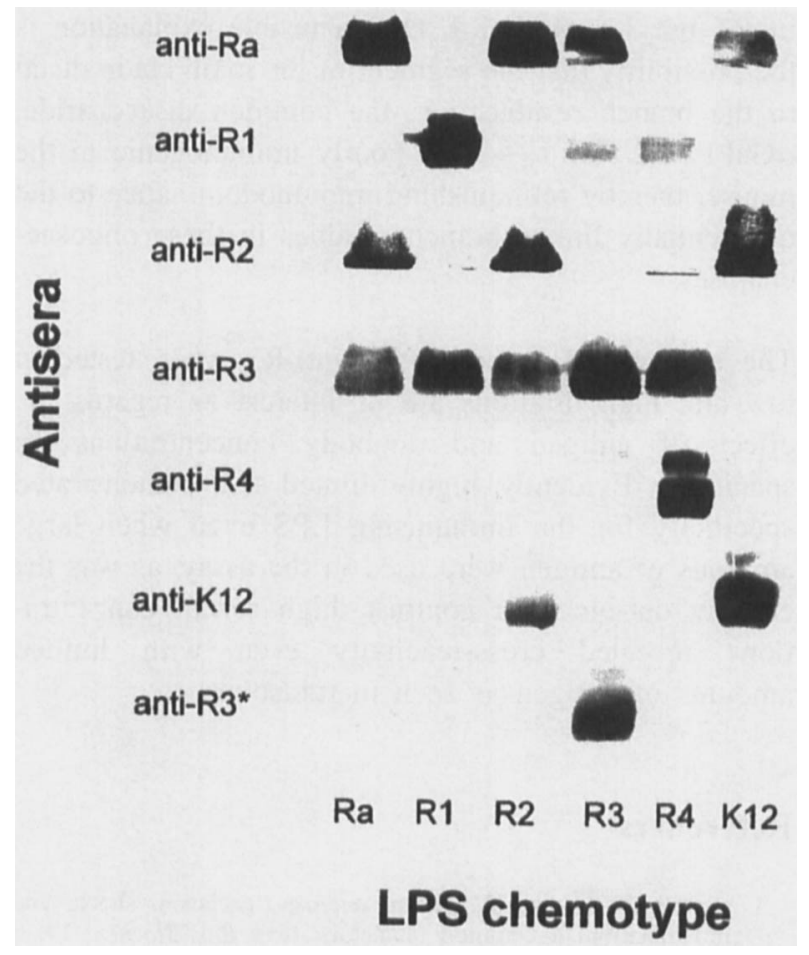

Fig. 6. Immunoblotting of anti-core sera against complete core chemotypes after resolution by SDS-PAGE.

mainly by immunisation with rough mutants of the Rc and Re chemotypes. Although these mutants have been reported to protect against infection with virulent heterologous strains [29-33], it remains to be estab-

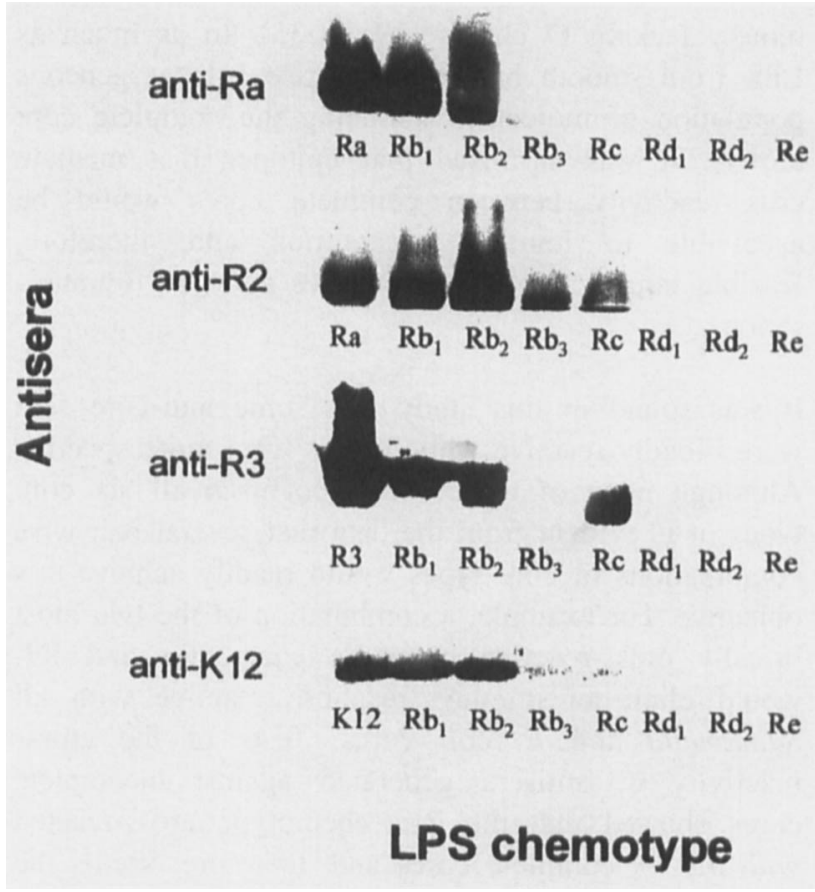

Fig. 7. Immunoblotting of five anti-core sera against various incomplete cores after resolution by SDS-PAGE.

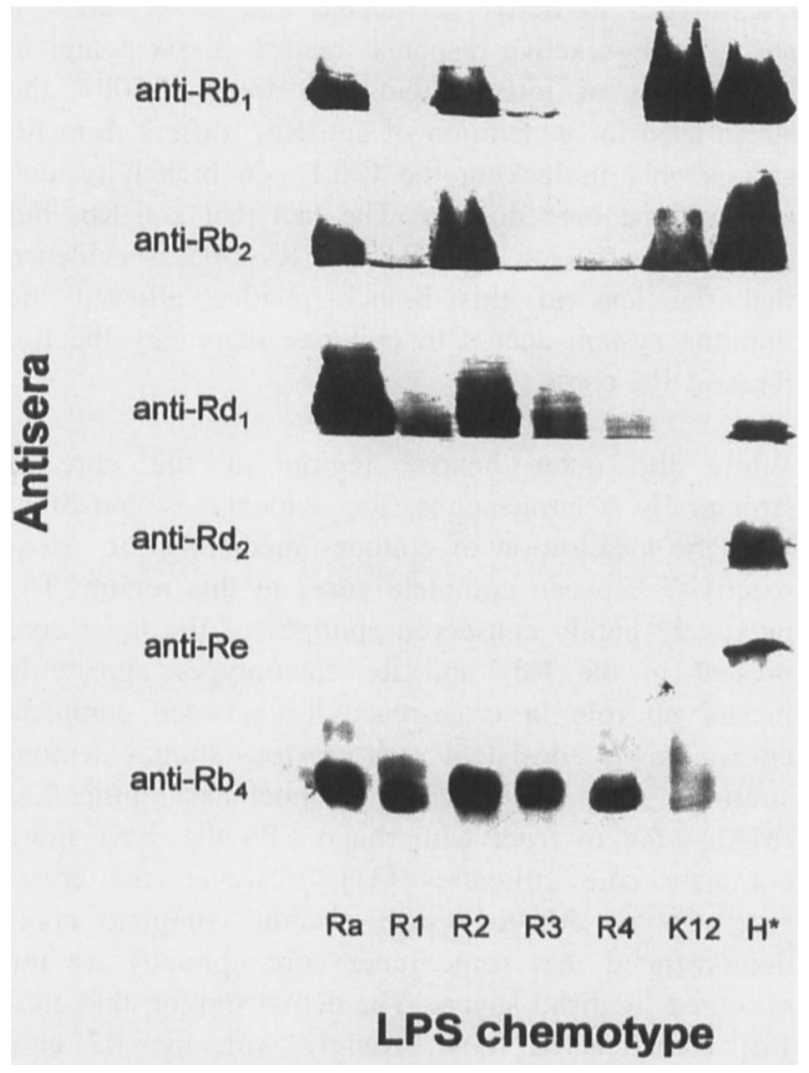

Fig. 8. Immunoblotting of antisera generated with incomplete cores against complete cores resolved by SDSPAGE. LPS antigens are designated by chemotype. Antigen $\mathrm{H}^{*}$ refers to the LPS chemotype homologous with the antiserum being tested.

lished that anti-LPS responses played a major role in the observed cross-protection [8]. On the contrary, several reports have suggested that inner core epitopes are inaccessible to antibodies even in the complete core 
moiety lacking $O$ chains $[19,34,35]$. In as much as LPS from smooth bacteria comprise a heterogeneous population of molecules including the complete core moiety, it was surmised that epitopes that mediate cross-reactivity between complete cores would be accessible to immune recognition and, therefore, feasible targets for cross-protective anti-LPS immunisation.

It was found in this study that some anti-core sera were broadly reactive while others were quite specific. Although none of these sera recognised all six core types, it is evident from the data that several pair-wise combinations of core types would readily achieve this objective. For example, a combination of the two most broadly cross-reactive complete cores, $\mathrm{Ra}$ and $\mathrm{R} 3$, would elicit an immune response reactive with all Salmonella and E. coli cores. Tests of the crossreactivity of antisera generated against incomplete cores showed that the $\mathrm{Rb}_{4}$ chemotype cross-reacted with all six complete cores and, therefore, seems the best chemotype for cross-reactive immunisation. As this chemotype cross-reacted only weakly with $\mathrm{K} 12$ LPS, it may be necessary to combine the two chemotypes to derive a vaccine that would elicit a strong cross-reactive response against all six complete cores. It is of interest that LPS from SL5007, the strain used for generation of anti- $\mathrm{Rb}_{4}$, differs from $\mathrm{Ra}$ strains only in lacking the Gal $1 \rightarrow 6$ branch residue (VII) of the core domain. The fact that anti- $\mathrm{Rb}_{4}$ but not anti-Ra reacted with R1 and R4 LPS is evidence that the loss of this branch residue allowed the immune system access to epitopes shared by the Ra, $\mathrm{R} 1$ and $\mathrm{R} 4$ cores.

While the outer hexose region of the core is structurally heterogeneous, the evidence is consistent with the localisation of epitopes mediating the crossreactivity between complete cores in this region. The putatively highly conserved epitopes of the inner core present in the $\mathrm{Rd}_{2}$ and $\mathrm{Re}$ chemotypes apparently played no role in cross-reactivity between complete cores. This is consistent with previous studies demonstrating that Re-specific monoclonal antibodies (MAbs) fail to react with rough LPS that have more complete core structures [31]. However, the crossreactivity of anti- $\mathrm{Rd}_{1}$ with several complete cores demonstrated that some inner core epitopes are not obscured by distal sugars. The demonstration that anti$\mathrm{Rd}_{1}$ cross-reacted most strongly with the $\mathrm{R} 2$ core agrees with a previous finding that a MAb specific for the inner core disaccharide, L- $\alpha$-D-heptose, $1 \rightarrow 7$-L- $\alpha$ D-heptose, of the Ra core cross-reacted with the R2 but not other cores [17]. Other investigators have also reported evidence for structural heterogeneity in the inner core involving differences in the numbers of heptose residues as well as in their substitution by phosphate and 2-aminoethyl pyrophosphate moieties [21]. It is of interest that antisera raised against complete cores did not recognise $\mathrm{Rd}_{1}$ LPS. This suggests that non-reciprocal recognition can result from differences between the steric/structural requirements for activation of immune responses versus those required for initiation of such effector functions as antibody binding. Thus, while the cross-reactive epitope associated with the $\operatorname{Rd}_{1}$ chemotype may have been too hidden to elicit antibodies when the Ra strain was used as immunogen, it was readily recognised within Ra LPS once specific antibodies had been generated by immunisation with the $\mathrm{Rd}_{1}$ strain.

The structural bases for cross-reactivity between complete cores are of interest. That between the $\mathrm{Ra}$ and $\mathrm{R} 2$ cores is readily explained by the fact that these cores demonstrate a high degree of structural similarity differing only in a single sugar residue (III) within the hexose region. The cross-reactivity between $\mathrm{K} 12$ and R2 LPS as evident in immunoblots of electrophoretically resolved LPS can also be explained similarly. However, the finding that the R1 and R4 cores were mutually poorly cross-reactive was unexpected in that these two cores are highly similar in structure within the hexose region. They share a common main chain tetrasaccharide, $\alpha$ Gall $\rightarrow-2-\alpha$ Gal $1 \rightarrow 2-\alpha$ Glc $1 \rightarrow 3-$ $\alpha \mathrm{Glc} 1 \rightarrow 3$, and differ in the hexose region only because a $\beta$ Glc branch residue (VIII), is linked $1 \rightarrow 3$ in $R 1$ but $1 \rightarrow 4$ in $R 4$. One plausible explanation is the possibility that the segment of the main chain distal to the branch residue, i.e., the common disaccharide, $\alpha \mathrm{Gal} 1 \rightarrow 2 \alpha \mathrm{Gal} 1 \rightarrow 2$, is poorly immunogenic in the mouse, thereby relinquishing immunodominance to the differentially linked branch residues in these oligosaccharides.

The recognition properties of anti-R3 when tested at low and high dilutions are of interest as regards the effect of antigen and antibody concentrations on specificity. Evidently, highly diluted sera demonstrated specificity for the immunising LPS even when large amounts of antigen were used in the assay, as was the case in dot-blots. In contrast, high serum concentrations revealed cross-reactivity even with limited amounts of antigen as seen in transblots.

\section{References}

1. Beutler B, Cerami A. Tumor necrosis, cachexia, shock, and inflammation: a common mediator. Annu Rev Biochem 1988; 57: 505-518.

2. Chedid L, Parant M, Parant F, Boyer F. A proposed mechanism for natural immunity to enterobacterial pathogens. $J$ Immunol 1968; 100: 292-301.

3. Lyman MB, Stocker BAD, Roantree RJ. Evaluation of the immune response directed against the Salmonella antigenic factors O4,5 and O9. Infect Immun 1979; 26: 956-965.

4. McCabe WR. Immunization with $\mathrm{R}$ mutants of $S$. minnesota. I. Protection against challenge with heterologous Gram-negative bacilli J Immunol 1972; 108: 601-610.

5. Muotiala A, Hovi M, Mäkelä PH. Protective immunity in mouse salmonellosis: comparison of smooth and rough live and killed vaccines. Microb Pathog 1989; 6: 51-60.

6. Nys M, Cloes JM, Demonty J, Joassin L. Protective effects of polyclonal sera and of monoclonal antibodies active on 
Salmonella minnesota Re595 lipopolysaccharide during experimental endotoxemia. $J$ Infect Dis 1990; 162: 1087-1095.

7. Ziegler EJ, McCutchan JA, Fierer J et al. Treatment of Gramnegative bacteremia and shock with human antiserum to a mutant Escherichia coli. $N$ Engl J Med 1982; 307: 1225-1230.

8. Greissman SE, Johnston CA. Evidence against the hypothesis that antibodies to the inner core of lipopolysaccharides in antisera raised by immunization with enterobacterial deeprough mutants confer broad-spectrum protection during gramnegative bacterial sepsis. $J$ Endotox Res 1977; 4: 123-153.

9. Ng A-K, Chen LH, Chang C-M, Nowotny A. Relationship of structure to function in bacterial endotoxins: serologically cross-reactive components and their effect on protection of mice against some gram-negative infections. $J$ Gen Microbiol 1976; 94: $107-116$.

10. Peter G, Chernow M, Keating MH, Ryff JC, Zinner SH Limited protective effect of rough mutant antisera in murine Escherichia coli bacteremia. Infection 1982; 10: 228-232.

11. van Dijk WC, Verbrugh HA, van Eme-van der Tol ME, Peters R, Verhoef J. Escherichia coli antibodies in opsonisation and protection against infection. $J$ Med Microbiol 1981; 14 381-389.

12. Bennett-Guerro E, Ayuso L, Hamilton-Davies $\mathrm{C}$ et al. Relationship of preoperative antiendotoxin core antibodies and adverse outcomes following cardiac surgery. JAMA 1997; 277: $646-650$.

13. Freeman R, Gould FK. Prevention of fever and gram negative infections after open heart surgery by antiendotoxin. Thorax 1985; 40: 846-848.

14. Goldie AS, Fearon KCH, Ross JA et al. Natural cytokine antagonists and endogenous antiendotoxin core antibodies in sepsis syndrome. JAMA 1995; 274: 172-177.

15. Gould FK, Harvey JA, Dytrych JK. Antibody to endotoxin is associated with decreased frequency of postoperative infection. Am J Obstet Gynecol 1989; 160: 317-319.

16. Hamilton-Davies C, Barclay GR, Machin SJ, Webb AR Relationship between endotoxin immune status and outcome following cardiac valve surgery. Br J Anaesth 1995; 74 Suppl: S34.

17 Nnalue NA, Lind SM, Lindberg AA. The disaccharide L- $\alpha-\mathrm{D}-$ Heptose $1 \rightarrow 7-L-\alpha$-D-Heptose $1 \rightarrow$ of the inner core domain of Salmonella lipopolysaccharide is accessible to antibody and is the epitope of a broadly reactive monoclonal antibody. J Immunol 1992; 149: 2722-2728.

18. Di Padova FE, Brade H, Barclay GR et al. A broadly crossprotective monoclonal antibody binding to Escherichia coli and Salmonella lipopolysaccharides. Infect Immun 1993; 61: 3863-3872.

19. Rietschel ETh, Seydel U, Zahringer U et al. Bacterial endotoxin: molecular relationship between structure and activity. Infect Dis Clin North Am 1991; 5: 753-779.

20. Jansson P-E, Lindberg AA, Lindberg B, Wollin R. Structural studies of the hexose region of the core in lipopolysaccharides from Enterobacteriaceae. Eur J Biochem 1981; 115: 571-577.

21. Holst $\mathrm{O}$, Brade $\mathrm{H}$. Chemical structure of the core region of lipopolysaccharides. In: Morrison DC, Ryan JL (eds) Bacterial endotoxin lipopolysaccharides, vol I. Molecular biochemistry and cellular biology. Boca Raton, CRC Press. 1992; 135-170.

22. Nnalue NA, Lindberg AA. Salmonella choleraesuis strains deficient of $\mathrm{O}$ antigen remain fully virulent for mice by parenteral inoculation but are avirulent by oral administration. Infect Immun 1990; 58: 2493-2501.

23. Wilkinson RG, Gemski P, Stocker BAD. Non-smooth mutants of Salmonella typhimurium: differentiation by phage sensitivity and genetic mapping. $J$ Gen Microbiol 1972; 70: 527-554.

24 Westphal O, Jann K. Bacterial lipopolysaccharides: extraction with phenol-water and further applications of the procedure. Methods Carbohydr Chem 1969; B5: 80-91.

25. Galanos C, Lüderitz O, Wesphal O. A new method for the extraction of R lipopolysaccharides. Eur J Biochem 1969; 9: 245-249.

26. Galanos C, Lüderitz O. Electrodialysis of lipopolysaccharides and their conversion to uniform salt forms. Eur J Biochem 1975; 54: 603-610.

27. Freudenberg M, Fomsgaard A, Mitov I, Galanos C. ELISA for antibodies to lipid A, lipopolysaccharides and other hydrophobic antigens. Infection $1989 ; 17: 322-328$.

28. Tsai C-M, Frasch CE. A sensitive silver stain for detecting lipopolysaccharides in polyacrylamide gels. Anal Biochem 1982; 119: 115-119.

29. Dunn DL, Ferguson RM. Immunotherapy of gram-negative bacterial sepsis: enhanced survival in a guinea pig model by use of rabbit antiserum to Escherichia coli J5. Surgery 1982; 92: 212-219.

30. Marks MI, Ziegler EJ, Douglas H, Corbeil LB, Braude AI. Induction of immunity against lethal Haemophilus influenzae type b infection by Escherichia coli core lipopolysaccharide. $J$ Clin Invest 1982; 69: 742-749.

31. Lind SM, Carlin NIA, Lindberg AA. Production and characterization of KDO-specific monoclonal antibodies recognizing lipopolysaccharides from heptoseless mutants of Salmonella. FEMS Microbiol Lett 1985; 28: 45-49.

32. Young LS, Stevens P, Ingram J. Functional role of antibody against "core" glycolipid of Enterobacteriaceae. J Clin Invest 1975; 56: 850-861.

33. Ziegler EJ, McCutchan JA, Douglas H, Braude AI. Prevention of lethal pseudomonas bacteremia with epimerase deficient $E$. coli antiserum. Trans Assoc Am Physicians 1975; 88: 101-108.

34. Siber GR, Kania SA, Warren HS. Cross-reactivity of rabbit antibodies to lipopolysaccharides of Escherichia coli J5 and other gram-negative bacteria. J Infect Dis 1985; 152: 954-964.

35. Shenep JL, Gigliotti F, Davis DS, Hildner WK. Reactivity of antibodies to core glycolipid with gram-negative bacteria. Rev Infect Dis 1987; 9 Suppl 5: S639-S643. 